\title{
Sol-Gel Derived Sensitive Films for Ammonia Sensors
}

\begin{abstract}
C. Tyszkiewicz*, P. Karasiński And R. Rogoziński
Department of Optoelectronics, Silesian University of Technology, Krzywoustego 2, 44-100 Gliwice, Poland

The sol-gel technology allows the preparation of silica thin films ranging in porosity from dense to highly porous films (50\%). Porous silica films can function as a matrix that is able to bind the molecules of pH-sensitive indicators and therefore can be utilized as a sensitive film in construction of intensity based fibre or planar optical sensors for ammonia detection. We report the fabrication and characterization of ammonia sensitive, highly porous silica films deposited on soda-lime glass plates and doped with the bromocresole purple, and bromothymol blue molecules. In this paper we present the influence of the substrate withdrawal speed from sol on a thickness and a refractive index of the undoped silica films, as well as spectral absorbance characteristics of the doped films. An influence of ammonia concentration in air and relative humidity on a transmission of produced sensitive films is also presented.
\end{abstract}

PACS numbers: 81.20.Fw, 42.70.Ce, 07.07.Df

\section{Introduction}

Ammonia is a naturally occurring chemical in the atmosphere. An average person detects ammonia by odor at $17 \mathrm{ppm}$. According to the WHO maximal admissible concentration allowed for people over 8 houres is $25 \mathrm{ppm}$ and over $10 \mathrm{~min} 35 \mathrm{ppm}$. Without protective equipment, the maximum airborne concentration below which it is believed that nearly all individuals could be exposed for up to $30 \mathrm{~min}$ without experiencing or developing life-threatening health effects is $500 \mathrm{ppm}$. Ammonia is present in the atmosphere due to natural biochemical processes and human activities (animal breeding, chemical industry, motorization). The measurement of ammonia concentration in air and water is important in agricultural industry, chemical industry and medical diagnosis in wide range of concentrations [1]. Because an instrumental analysis is expensive and time-consuming the new methods for determination of ammonia concentration are being developed. Among them are methods utilizing SAW gas sensors [2] and optical sensors. The optical methods base on sensitive films which are able to change their absorbance [3-5] or upconversion luminescence phenomena in films comprising upconverting nanoparticles (UCNPs) [6] due to a presence of ammonia. Among the materials belonging to the first group metioned above is a polyaniline and $\mathrm{pH}$-indicators. The physical effect occurring in a sensitive film determines the applicable optical transducer. Absorption changes of the sensitive films can be detected by the waveguiding sensor structures using evanescent wave spectroscopy. In such structures the propagating optical wave penetrates the sensitive film with its evanescent wave. Therefore absorption changes in the sensitive layer causes changes in amplitude of the propagating waveguide modes (light intensity changes). Optical sensors can be fabricated using optical fibres $[7,8]$ or planar waveguides [9-14]. An advantage of the sensor structures exploiting the optical

* corresponding author; e-mail: cuma.tyszkiewicz@polsl.pl fibres used in telecommunication industry is a capability of using standarized optical fibre devices (couplers, detectors). An application of the planar waveguides allows for an optimization of optical and geometrical parameters of the sensor structure in wide range $[16,17]$.

Presented work regards the sensing films for optical waveguide ammonia sensors exploiting evanescent wave spectroscopy. The films are composed of porous silica which is a matrix bonding ammonia sensitive $\mathrm{pH}-$ -indicator. Such films method of low porosity $(P \approx 7 \%)$, fabricated using sol-gel method, were earlier investigated in our research group [18] and appplied in planar amplitude ammonia sensor [19]. Among the advantages of the sol-gel method is having control over the structure of the fabricated material, especially porosity [20]. Solgel method allows the fabrication of porous silica films characterized by the refractive index much lower than solid silica. Now we fabricated such films of porosity $P \approx 50 \%$ (refractive index $n \approx 1.2$ ). The porous silica films were sensitized to ammonia by means of impregnation with bromocresole purple and bromothymole blue. Adopted fabrication method allow for optimization of refractive index and thickness parameters of silica layer and pH-indicator concentration. However, optimization cannot be carried out in isolation from the parameters of the applied waveguide being a part of the optical transducer [17]. In this work there are presented the spectral absorbance characteristics of the fabricated sensitive films and the results of the investigations on their sensing properties toward ammonia.

\section{Technology}

Sol-gel method allows for chemical fabrication of glasses and ceramics from a liquid phase. The sol-gel process can be split into the following subsequent stages: formation of the colloidal system, hydrolysis and condensation, deposition of a sol layer on the substrate, drying and annealing of the fabricated film [20]. In technological processes that led to fabrication of the sensing films presented in this paper the precursor of silica was tetraethyl orthosilicate $\mathrm{Si}\left(\mathrm{OC}_{2} \mathrm{H}_{5}\right)_{4}$. Ethanol $\mathrm{C}_{2} \mathrm{H}_{5} \mathrm{OH}$ was used 
as a solvent and hydrochloric acid as a catalyst. The molar ratio of initial components and the amount of catalyst as well as parameters characterizing course of each stage in sol-gel process have the influence on parameters of the fabricated film. The prime advantage of the sol-gel method is a capability of controlling the structure of fabricated material. The other advantages are: high uniformity of fabricated films, low temperature of the fabrication processes and capability of different material formation (films, fibres, powders). The sol-gel based films of high porosity can be used for fabrication the sensitive films which are a part of the amplitude-type chemical sensors exploiting evanescent wave spectroscopy. The porous silica functions as a matrix that bounds the $\mathrm{pH}-$ -indicators. The sensing properties of such films are governed by their porosity and $\mathrm{pH}$-indicator type. The values of porosity have an influence on their responsiveness and regeneration time. High porosity of the fabricated layers was achieved by adding a nonpolar surface agent to the sol. The agent reduces the surface tension between a solution and a substrate and an internal tension occurring on the condensation stage leads to contraction and material structure densification.

The silica films were deposited on glass substrates using the dip-coating method. The thickness of fabricated films was controlled through the withdrawal speed of the glass substrate from the sol. Sol films deposited on substrates were dried and annealed for $40 \mathrm{~min}$ at $425^{\circ} \mathrm{C}$. The fabricated structures were impregnated with bromocresole purple (BP) and bromothymole blue (BB) by immersion in ethanol based solutions of each $\mathrm{pH}$-indicator. The $0.001 \mathrm{~mol}$ of each $\mathrm{pH}$-indicator was diluted in $100 \mathrm{ml}$ of $\mathrm{EtOH}$ forming the solution in which the structures were impregnated for $10 \mathrm{~min}$. After impregnation the structures were dried for $15 \mathrm{~min}$ at $90^{\circ} \mathrm{C}$.

\section{Measuring methods}

The investigations on the fabrication of the sensitive films were broken down into three stages. The first stage involved the fabrication technology of porous silica films on glass substrates. The thickness and refractive index of the films were measured with a monochromatic ellipsometer Sentech SE400 $(\lambda=632.8 \mathrm{~nm})$.

In the second stage spectral absorbance characteristics of porous silica films and sensitive films were carried out using Ocean Optics HR4000CG UV-VIS spectrophotometer. The range of wavelengths over which the spectra were registered is $190-1100 \mathrm{~nm}$ with resolution $0.75 \mathrm{~nm}$. The measurement setup is shown in Fig. 1. Mikropack DT-MINI-2-GS was used as wideband light source. First the transmission spectrum $T_{0}(\lambda)$ of porous silica film was measured. In order to determine the changes in absorbance triggered by ammonia two additional spectra were measured: $T_{\mathrm{I}}(\lambda)$ of sensitized porous silica film and $T_{\mathrm{IZ}}(\lambda)$ of sensitized porous silica film subjected to ammonia.

The spectral absorbance characteristics allow us to determine the wavelength of maximal absorption in the

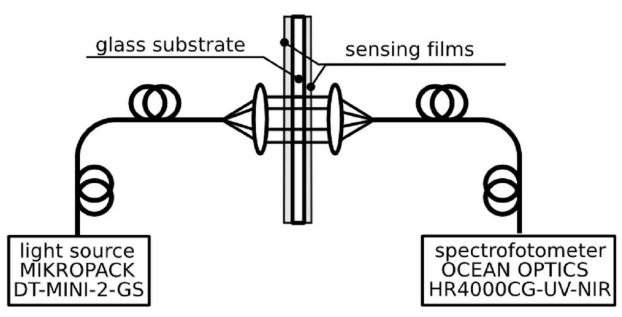

Fig. 1. Experimental setup for determination of the spectral transmission characteristics.

presence of ammonia. The source of ammonia for these measurements was a $25 \%$ solution of ammonium hydroxide.

In the third stage the sensitive films were subjected to the mixture of ammonia in synthetic air of controlled ammonia concentration. The temperature and relative humidity of synthetic air was also controlled. On the basis of relative humidity and temperature measurements the concentration of water vapour expressed in ppm was determined on the basis of the updated Arden-Buck equation [21]. The measurement setup is shown in Fig. 2.

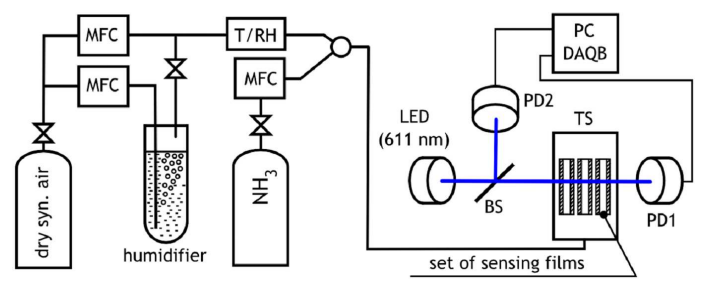

Fig. 2. Experimental setup for determination of the transmission characteristics at optimized wavelength. MFC - mass flow controller, BS - beam splitter, TS — test chamber, PD1 - photodiode, PD2 - reference photodiode, $\mathrm{T} / \mathrm{RH}$ - relative humidity and temperature sensor.

The structures were grouped in fours in measuring chamber. As a light source there was used a LED diode operating at wavelength $\lambda=611 \mathrm{~nm}$ which is closest to the wavelength of maximal absorbance of both $\mathrm{pH}$ -indicators. The photodiode PD1 have measured the intensity of light passing through the sensitive films. The reference photodiode PD2 have measured the changes in optical power emitted from LED.

\section{Experimental results}

A main parameter of the dip-coating method which allows control over film thickness is the withdrawal speed of the substrate from sol. In Fig. 3 there is shown the characteristics of the influence of substrate withdrawal speed $v$ from sol on silica film thickness $d$ and refractive index $n$. For substrate withdrawal speed in range $v=3.06-7.27 \mathrm{~cm} / \mathrm{min}$ fabricated films thickness is in range $d=537-906 \mathrm{~nm}$. The increase in withdrawal speed 
results in decrease of the film refractive index. Such a relationship is typical for polymeric sols in case of weakly charged polymers and high condensation speed [20]. In case shown in Fig. 3 the relationship between refractive index $n$ and withdrawal speed $v$ is given by equation

$$
n=1.2387(20)-0.0013(4) v .
$$

Low refractive index is a consequence of silica film high porosity. The porosity can be estimated using the approximated equation for effective refractive index of the medium: $n=n_{\text {air }} P+n_{\mathrm{d}}(1-P)$, where $n_{\text {air }}=1$ is a refractive index of air and $n_{\mathrm{d}}=1.4585$ is refractive index of solid silica at wavelength $\lambda=632.8 \mathrm{~nm}$. The porosity $P$ of fabricated films is approximately 0.5 .

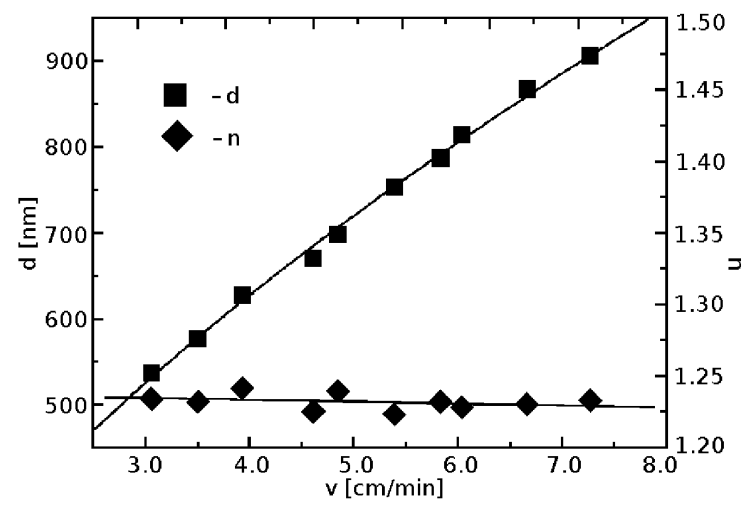

Fig. 3. Influence of substrate withdrawal speed from sol on silica film thickness and refractive index $(\lambda=$ $632.8 \mathrm{~nm})$.

Transmission characteristics of fabricated films against a background of transmission characteristic of soda-lime glass substrate are presented in Fig. 4. The curve A shows the transmission of soda-lime glass substrate sandwiched between two porous silica films. The thickness of each film is $d=906 \mathrm{~nm}$. Well defined interference maxima and minima are visible. Moreover it can be seen that the substrate coated on both sides has better transmission characteristics than the uncoated one. The location of interference minima below the substrate level is caused by a non-uniformity of porous silica film refractive index. This non-uniformity results from a stress raised during drying and annealing of silica films. The curve B matches the same structure after impregnation in the solution of BP. This characteristic was registered in air. It can be seen that a presence of the BP induces absorption at the wavelength $\lambda=422 \mathrm{~nm}$. For the wavelengths exceeding $\lambda=540 \mathrm{~nm}$ the presence of BP does not change transmission. The curve $\mathrm{C}$ was registered for the above mentioned structure influenced by ammonium hydroxide vapours from the $25 \%$ solution of ammonium hydroxide. The curve D was registered one minute after start of regeneration of the structure in air.

Spectral characteristics of absorbance difference $\Delta A$ of sensitive films based on BP and BB are shown on the left plot in Fig. 5. The $\Delta A$ was calculated using the following

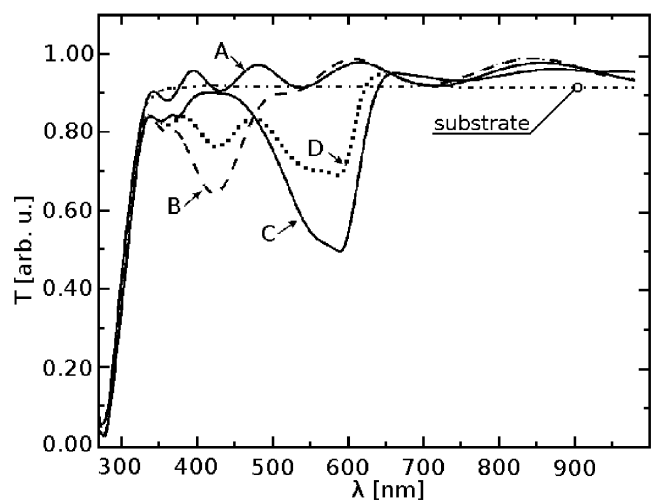

Fig. 4. Spectral transmission characteristics of the structure composed of two BP-based sensitive films.

equation:

$$
\Delta A=\log \left(\frac{T_{\mathrm{I}}}{T_{\mathrm{IZ}}}\right),
$$

where $T_{\mathrm{I}}$ is a transmission characteristic of sensitized porous silica film and $T_{\mathrm{IZ}}$ is a transmission characteristic of the same film subjected to ammonia.

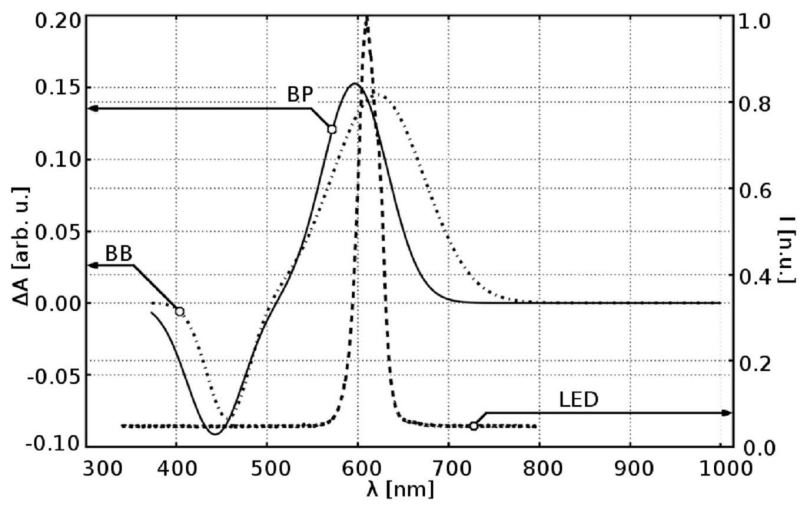

Fig. 5. Spectral characteristics of change in absorbance of the sensitive films with $\mathrm{BP}$ and $\mathrm{BB}$ compared to the spectral characteristic of the LED.

Calculated spectral characteristics of absorption coefficient $\alpha$ and extinction coefficient $n^{(i)}$ for bromocresole purple based sensitive films are shown in Fig. 6. On the bases of the spectral absorbance characteristics a LED was chosen for which absorbance of each $\mathrm{pH}$-indicator is the highest possible.

Transmission characteristics of sensitive films subjected to $\mathrm{NH}_{3}$ of controlled concentration were obtained using the measuring setup shown in Fig. 2. The signals from both photodiodes were normalized against the maximal value in each signal. In order to obtain the normalized transmission $T$ independent of LED optical power drift the signal from PD1 was divided by the reference signal from PD2. The structures were gathered into two groups. Each group composed of four structures impregnated with the same $\mathrm{pH}$-indicator was placed in the test 


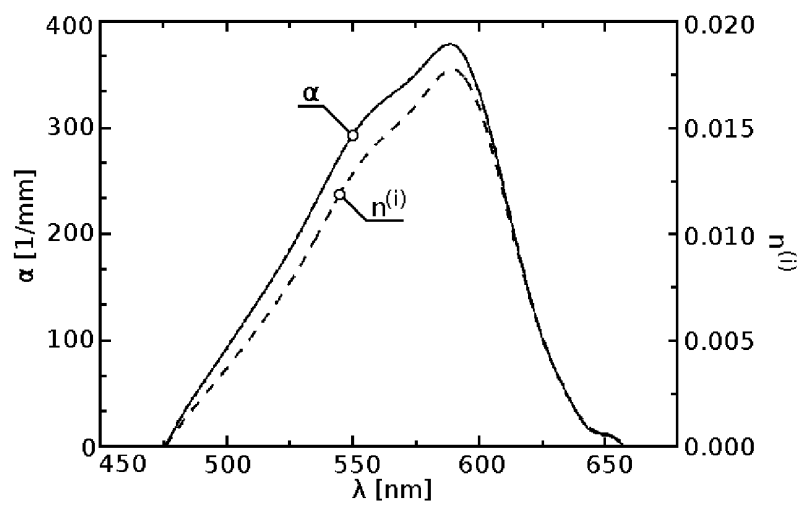

Fig. 6. Spectral characteristic of absorption coefficient and extinction coefficient for BP-based films.

chamber.

In the first stage the structures were subjected to four exposure sequences of target gas diluted in unhumidified air. Each of these exposure sequences consisted of $1200 \mathrm{~s}$ of purge and $1200 \mathrm{~s}$ of exposure to $491 \mathrm{ppm}$ of $\mathrm{NH}_{3}$. The structures were exposed during the purge subsequence to unhumidified synthetic air. The results for BP based structures and BB based ones are shown in Fig. 7 and Fig. 8, respectively. It can be seen that the signal in each exposure is reproducible. The slow regeneration of the sensitive films during the purge is caused by the relatively high volume of the test chamber $\left(V=90 \mathrm{~cm}^{3}\right)$ and slow total flow $(f=200 \mathrm{sccm})$. The transmission drop of BP-based structures is $\approx 2.75$ times higher than in case of the BB-based samples.

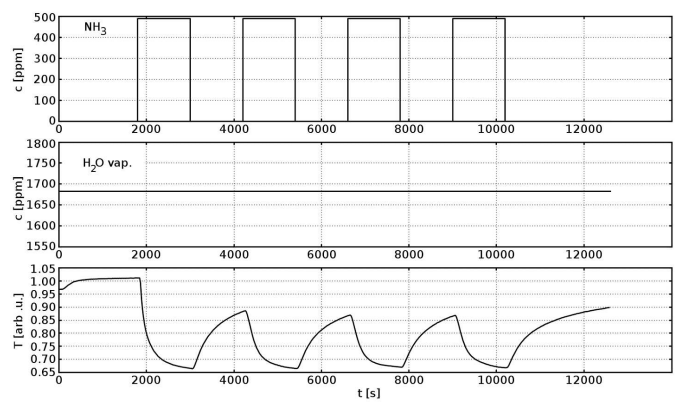

Fig. 7. Transmission characteristic of BP-based sensitive films subjected to $\mathrm{NH}_{3}$ of constant concentration $c=491 \mathrm{ppm}$.

In the second stage the structures were subjected to six exposure sequences for BP-based structures and five for BB-based structures of target gas diluted in humidified air. Each of these exposure sequences consisted of $1200 \mathrm{~s}$ of purge and $1200 \mathrm{~s}$ of exposure to $245 \mathrm{ppm}$ of $\mathrm{NH}_{3}$. In subsequent exposures to target gas the water vapour concentration raised.

The structures were exposed during the purge subsequences to unhumidified synthetic air. The results for bromocresole purple based structures and bromothymole

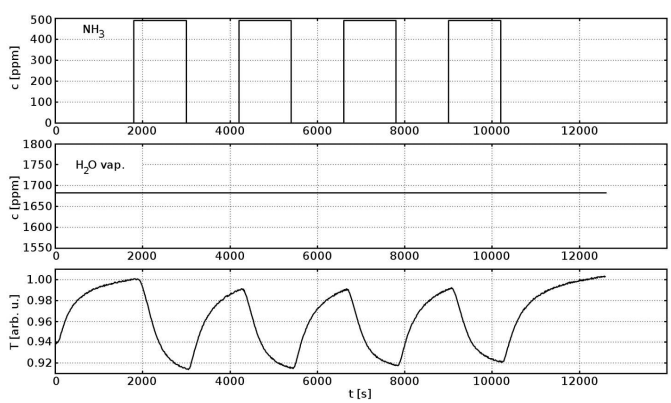

Fig. 8. Transmission characteristic of BB-based sensitive films subjected to $\mathrm{NH}_{3}$ of constant concentration $c=491 \mathrm{ppm}$.

blue based ones are shown in Fig. 9 and Fig. 10, respectively. The increase in the concentration of water vapour causes the increase in transmission drop at the constant concentration of $\mathrm{NH}_{3}$.

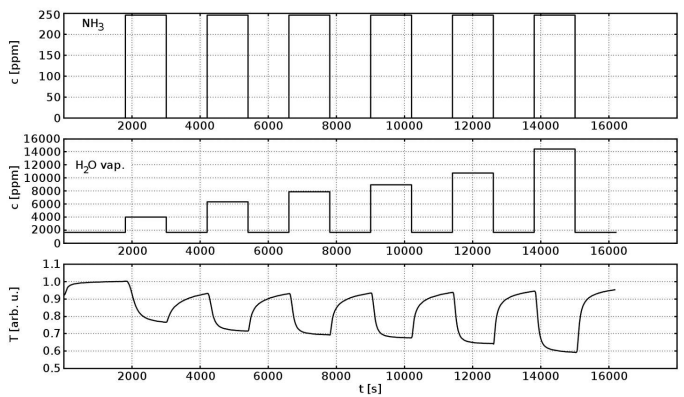

Fig. 9. Transmission characteristic of BP-based sensitive films subjected to $\mathrm{NH}_{3}$ of constant concentration $c=250 \mathrm{ppm}$ and increasing concentration of the water vapour.

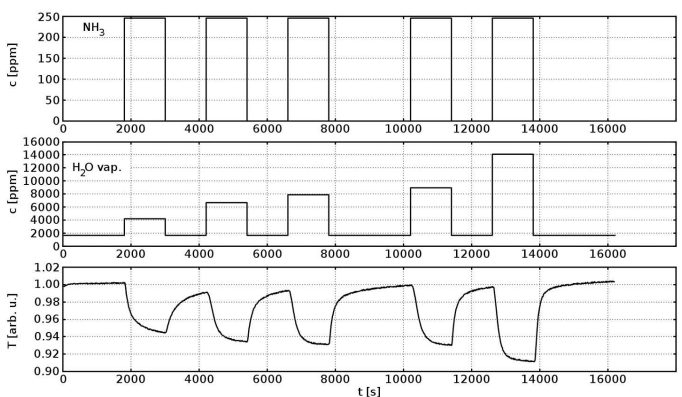

Fig. 10. Transmission characteristic of BB-based sensitive films subjected to $\mathrm{NH}_{3}$ of constant concentration $c=250 \mathrm{ppm}$ and increasing concentration of the water vapour.

In the third stage the structures were subjected to nine exposure sequences of target gas diluted in humidified air. Each of these exposure sequences consisted of $4000 \mathrm{~s}$ of purge and $1800 \mathrm{~s}$ of exposure to $\mathrm{NH}_{3}$. In the first four exposures the ammonia concentration increased in 
steps from $49 \mathrm{ppm}$ to $245 \mathrm{ppm}$ and the concentration of water vapour decreased. In the subsequent five exposures the concentration of ammonia and water vapour was constant. The structures were exposed during the purge to humidified synthetic air of constant concentration of water vapour. The results for BP-based structures and BB-based ones are shown in Fig. 11 and Fig. 12, respectively. The first four exposures in this set show that the changes in $\mathrm{NH}_{3}$ concentration have stronger influence on absorption coefficient than changes in water vapour concentration because the transmission drops in spite of the decrease of water vapour concentration. The last five exposure sequences again proves the repetitiveness of the sensitive films response.

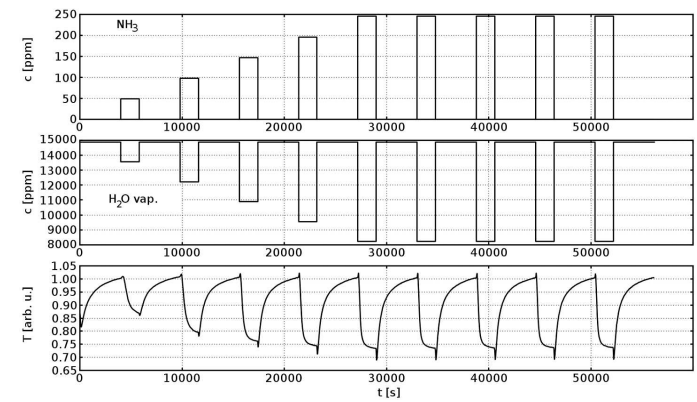

Fig. 11. Transmission characteristic of BP-based sensitive films subjected to ammonia of increasing concentration and decreasing concentration of the water vapour.

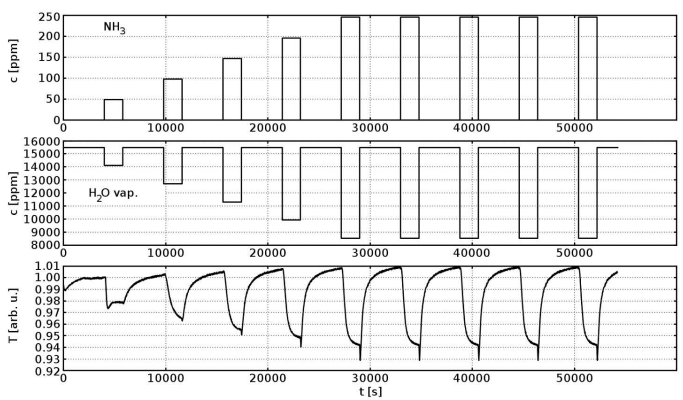

Fig. 12. Transmission characteristic of bromothymol blue (BB) based sensitive films subjected to ammonia of increasing concentration and decreasing concentration of the water vapour.

\section{Summary}

In this work the results of our investigation on fabrication technology and characterization of ammonia sensitive optical films are presented. Fabricated films can be applied in chemical optical waveguide sensors exploiting evanescent wave spectroscopy. Taking advantage of sol-gel technology, fabricated film are highly porous and function as a host matrix for $\mathrm{pH}$-sensitive indicators: $\mathrm{BP}$ and BB. Carried out investigations showed that for high concentration of ammonia an increase in absorption is comparable for films based on both indicators. However, for ammonia concentration range (50-500 ppm) the BP-based films are more sensitive. Moreover, the concentration of water vapour has significant effect on absorption of investigated films. Therefore temperature and relative humidity control is necessary. The investigations on regeneration time are not presented in the scope of this work. However, due to high porosity and low thickness (several hundred nanometres) of fabricated films we anticipate short regeneration times.

\section{Acknowledgments}

This work is partially sponsored by the Polish Ministry of Science and Higher Education within a scope of the grant of the NCBiR N R01 0034 06/2009.

\section{References}

[1] B. Timmer, W. Olthuis, A. Van Den Berg, Sensors Actuators B 107, 666 (2005).

[2] W.P. Jakubik, M. Urbańczyk, E. Maciak, T. Pustelny, Acta Phys. Pol. A 116, 315 (2009).

[3] A. Persad, K.-F. Chow, W. Wang, E. Wang, A. Okafor, N. Jespersen, J. Mann, A. Bocarsly, Sensors Actuators B 129, 359 (2008).

[4] V.M.N. Passaro, F. Dell'Olio, F. De Leonardis, Sensors 7, 2741 (2007).

[5] P. Karasiński, R. Rogoziński, C. Tyszkiewicz, Pomiary Akustyka Kontrola 56, 541 (2010).

[6] H.S. Mader, O.S. Wolfbeis, Anal. Chem. 82, 5002 (2010).

[7] S.K. Khijwania, B.D. Gupta, Opt. Quant. Electron. 31, 625 (1999).

[8] C. McDonagh, C.S. Burke, B.D. MacCraith, Chem. Rev. 108, 400 (2008).

[9] P.V. Lambeck, Meas. Sci. Technol. 17, R93 (2006).

[10] R. Klein, E. Voges, Sensors Actuators B 11, 221 (1993).

[11] P. Karasiński, R. Rogoziński, Proc. SPIE 5576, 186 (2004).

[12] Y.S. Lee, B.S. Joo, N.J. Choi, J.O. Lim, J.S. Huh, D.D. Lee, Sensors Actuators B 93, 148 (2003).

[13] A. Airoudj, D. Debarnot, B. Bêche, F. Pancin-Epaillard, Talanta 76, 314 (2008).

[14] M. Błahut, D. Kasprzak, Acta Phys. Pol. A 116, 261 (2009).

[15] P. Karasiński, R. Rogoziński, Proc. SPIE 4516, 218 (2001).

[16] P. Karasiński, R. Rogoziński, Opt. Commun. 269, 76 (2007).

[17] P. Karasiński, Opt. Appl. 33, 477 (2003).

[18] P. Karasiński, R. Rogoziński, Proc. SPIE 5576, 186 (2004).

[19] L.C. Klein, Sol-Gel Optics, Processing and Applications, Kluwer Academic Publishers, 1994.

[20] A.L. Buck, J. Appl. Meteorol. 20, 1527 (1981). 